\title{
SUR LES MESURES A PRENDRE EN VUE D'AUGMENTER LA CONSOMMATION DE LA CARPE, EN FRANCE
}

\author{
par RenÉ CHARPY \\ Conservateur des Eaux et Forets. \\ Chargé de la Pêche à la Direction générale des Eaux et Forêts.
}

La France dispose de 108.800 hectares d'étangs privés en état de rapport produisant environ 8.000 tonnes commercialisées par an soit sensiblement 73 kilos par hectare et par an. Cette production représente $46 \%$ de notre production totale en poissons d'eau douce évaluée à 17.560 tonnes.

En valeur, elle correspond approximativement à 1.620 .000 .000 de francs, soit $24 \%$ seulement de la valeur totale de notre production métropolitaine en poissons d'eau douce (6.730.000.000 de fr.).

Pourquoi cet écart important entre le pourcentage-tonnage $(46 \%)$ et le pourcentage-valeur (24\%) ? C'est que l'exploitation de nos étangs est essentiellement orientée vers la production de poissons d'une valeur commerciale assez faible, tels que Carpes, Tanches et incidemment Gardons blancs et Rotengles, qui représentent environ $90 \%$ de cette production, alors que $10 \%$ seulement portent sur des poissons de plus grande qualité : Brochets, et, en quantité beaucoup moindre, Black-bass.

La Carpe étant actuellement le poisson essentiel de nos étangs et la production de ceux-ci atteignant plus des $3 f 5^{\circ}$ de notre production métropolitaine en poissons d'eau douce, c'est à propos de la Carpe qu'il convient d'examiner les possibilités d'accroissement de notre consommation en poissons d'eau douce. Or, cette consommation est des plus modeste. Elle n'atteint, en effet, que 430 grammes par an et par habitant. Il y aurait donc intérêt à prendre toutes mesures en vue de l'accroître.

A cet effet, la seule propagande vraiment efficace, du moins dans la France métropolitaine, consisterait, semble-t-il, à mettre la Carpe à la portée du consommateur et à ne livrer à ce consommateur que des produits 
de qualité. Nous pensons en effet que les autres moyens de propagande : affiches, articles de journaux, radio, tracts, expositions, cours de cuisine, primes aux nouveaux débits, journées du poisson, création de restaurants dits " au poisson ", etc., usités dans certains pays de l'Europe Centrale, où ils sont d'ailleurs utiles parce que le poisson d'eau douce y tient déjá une place considérable sur le marché, nous paraissent chez nous absolument illusoires.

$\mathrm{Si}$, en France le marché intérieur de la Carpe était tant soit peu important il pourrait être fait appel aux moyens de propagande visés ci-avant, en vue d'accroître encore quelque peu ce marché. Mais tel n'est pas le cas; aussi estimons-nous qu'il n'y a pas lieu de recourir à ces moyens qui, à nos yeux, correspondent, pour ce qui nous concerne, à la loi du moindre effort. Ce n'est pas parce qu'à la radio on répètera aux Français : " mangez de la Carpe ", qu'ils en achèteront. Ce serait s'engager là dans des dépenses n'ayant aucune efficacité. Si l'on veut développer le marché intérieur de la Carpe ou pour ainsi dire le créer, il faut comme dit ci-avant, mettre la Carpe à la portée du consommateur et ne lui livrer que des produits de qualité.

Au surplus, si l'on examine quelles sont les régions de France où l'on consomme vraiment plus de poisson d'eau douce qu'ailleurs, on constate que ces régions sont les suivantes :

Paris, où les Halles Centrales disposent de vastes bassins alimentés par de l'eau courante et où certains mandataires possèdent des bacs pour y conserver les poissons vivants pendant parfois plusieurs semaines.

Lyon, centre, il est vrai, d'une région particulièrement riche en étangs (la Dombe) et en cours d'eau, où le poisson d'eau douce pêché à une distance relativement faible, est entreposé vivant dans des bacs aménagés sur les bords du Rhône, et alimentés avec l'eau de ce fleuve et où, par conséquent, le poisson est livré à la consommation dans un parfait état de fraîcheur.

Le Forez, où la Société Coopérative agricole des Carpes contrôlées du Forez a créé des viviers-dépôts de poissons à Marcigny, à Palinges, aux Louteaux près de Moulins, et fourni aux revendeurs des viviers flottants en bois placés dans les cours d'eau, soit même, à certains, des viviers en ciment armé (Autun, Digoin, Varennes-sur-Allier, etc.).

Certaines régions du sud-est de la France, où les magasins dits du "Casino », vendent dans leurs succursales multiples, à la fois de la marée et de la Carpe avec garantie syndicale, sans que l'une nuise à l'autre.

En somme, c'est bien dans les régions où la Carpe est mise à la portée du consommateur que ce poisson trouve preneur. 
Pour mettre le poisson à la portée du consommateur, il faut inévitablement organiser des centres de stockage dans les grandes régions de consommation et prévoir des moyens de distribution sous forme de bacs-viviers, chez les détaillants.

Ces centres de stockage peuvent être représentés par de grands viviers ou mieux par des péniches-viviers. Dès 1927, la Société Coopérative Agricole des Carpes contrôlées du Forez, avait ainsi envisagé l'aménagement d'une péniche-vivier à l'amont de Paris, et la livraison d'une Carpe-standard aux Halles, tous les jours, par 500 kilos (de façon à empêcher l'usage des faux cours). Cet aménagement fut sur le point d'être entrepris en 1939 par la Société en question, en accord avec le Syndicat de Contrôle des Éleveurs de Carpes. Ce projet serait à reprendre et à réaliser.

D'autres centres pourraient utilement être créés ailleurs, notamment dans le bassin de Longwy non loin des étangs de la Moselle, dans la vallée du Rhône pour les étangs du Forez, dans la région toulousaine, etc.

L'organisation de tels centres serait à financer pour moitié par l'État et pour l'autre moitié par les Coopératives des producteurs intéressés.

Dans bien des cas, il serait possible de prévoir des viviers urbains pouvant servir de piscines durant l'été et qui, de ce fait, pourraient être subventionnés par l'État, au double titre du ravitaillement et des sports, puis par les villes, puis, bien entendu, par les Coopératives.

Pour toucher le consommateur, il est enfin essentiel que des bacsviviers soient établis chez les distributeurs eux-mêmes. Ce système, qui est évidemment coûteux comme premier investissement, n'a été pratiqué jusqu'à présent, et ce avec prudence, que par le Syndicat des Étangs du Forez, mais il lui a donné des résultats supérieurs à tous les autres moyens de propagande essayés par lui. Pour l'étendre, il conviendrait de fabriquer du matériel en série, et de le louer, par l'intermédiaire notamment de l'Union Nationale des Syndicats de l'Étang, aux détaillants, à charge par eux de ne vendre que de la Carpe vivante et sélectionnée.

"Partout ", a écrit le Comte de Neufbourg, "où l'on a pu livrer aux épiciers, des Carpes et des Tanches calibrées, que le client choisit lui-même dans le bac, sans pesée, le poisson vif s'est vendu. La pesée est le grand dégoût du détaillant. Si l'on calibre honnêtement, si l'on vend au prix moyen du kilo, des Carpes d'au moins deux livres et d'au plus 1.100 grammes, sans tricherie, le public renonce à sa chère pesée n.

Ajoutons qu'il n'est pas indispensable de disposer de bacs alimentés en eau courante. L'installation de postes fixes avec canalisations, la consommation d'eau, etc. entraînent d'ailleurs des frais très importants et se heurtent à des impossibilités pour les boutiques ambulantes. Or, on arrive fort bien à conserver des poissons vivants, sans renouvelle- 
ment de l'eau, à la condition que cette eau soit aérée Pour ce faire, on dispose actuellement de petits compresseurs d'air qui, simplement branchés sur une canalisation de lumière électrique, peuvent rendre d'excellents services.

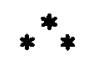

Mais il ne suffit pas, pour gagner la bataille du poisson d'eau douce, que le poisson trouve actuellement preneur. Il faut encore que la clientèle prenne goût à la Carpe, en garde un bon souvenir, et $\mathrm{y}$ revienne. En un mot, il faut songer à conserver le client. Il est dès lors nécessaire de ne lui livrer que des produits de qualité.

Ceci explique la nécessité de ne produire que de la Carpe sélectionnée, garantie, épaisse, charnue, ayant crû rapidement et présentant, de ce fait, le minimum d'arêtes.

Depuis déjà de nombreuses années, le Syndicat de contrôle de la Carpe d'élite impose à ses adhérents la production de Carpes présentant un indice de sélection, $I=\frac{L}{H}(1)$, inférieur à 2,8 , et ces Carpes s'écoulent toujours sans difficultés. Au Forez, par exemple, la consommation de la Carpe a quadruplé depuis que fonctionne la garantie syndicale. C'est donc dans ce sens qu'il faut travailler.

Comme l'a très bien dit le Comte de Neufbourg, dans une communication faite par lui au IIIe Congrès National de l'Étang, se rapportant il est vrai à l'exportation, «les pays qui contrôlent leurs produits et ne laissent exporter que des fruits, légumes, fromages, œufs, etc. garantis, ont accaparé les marchés dont nos produits ont disparu. La nécessité du contrôle de la Carpe est ainsi démontrée ". Or, pour la Carpe, qu'elle soit ou non pourvue d'écailles, ce qui importe avant tout, c'est que sa croissance soit rapide, afin que son squelette soit aussi réduit que possible, ses filets épais et sa chair pour ainsi dire sans arêtes.

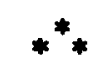

Mais, si la Carpe doit être de qualité, il faut aussi qu'elle soit bien dégorgée, d'où l'absolue nécessité pour les propriétaires et exploitants d'étangs, de disposer de viviers de dégorgement. Depuis déjà plus de 25 ans, le Comte DE Neufbourg répète que : " tout étang dépourvu de vivier est de demi-valeur ". Nous nous rallions pleinement à cette formule : le vivier est nécessaire pour y faire dégorger la Carpe (et aussi pour y conserver la Tanche après l'automne), assurer de la sorte un meilleur écoulement des pêches et éviter de ce fait que le commerce n'achète d'abord le poisson des producteurs négligents ou ignorants des cours, des procédés d'élevage et de vente.

(1) Rapport de la longueur du poisson, du museau à la naissance de la queue, sur sa plus grande hauteur. 
Cette nécessité du vivier s'impose tout particulièrement dans les régions de petits propriétaires, telle, pour prendre un exemple, la Brenne. Cette région qui est presque entièrement comprise dans l'arrondissement du Blanc (Indre), comporte 10.000 hectares d'étangs appartenant à environ 400 propriétaires. Or, si dans la Brenne, les grands propriétaires disposent de viviers importants, les autres, au nombre de 350 environ, n'en ont point. C'est là une situation d'autant plus fâcheuse que, par suite des conditions hydrologiques, tout au moins en année moyenne, on doit commencer très tôt à procéder au remplissage des étangs ; les pêches doivent donc avoir lieu durant les trois derniers mois de l'année et ce, sans possibilité de stockage, alors que la grande saison de consommation va de Février à Mars. Aussi, sauf celles à provenir des quelques viviers existants, de grandes quantités de poissons arrivent en même temps sur le marché, ce qui a pour conséquence de faire baisser les prix, au préjudice des exploitants; en outre, ce poisson n'est pas dégorgé.

Il serait donc très utile que les petits propriétaires d'étangs aient à leur disposition des viviers-réservoirs pour y entreposer le poisson avant sa mise en vente. La création de tels réservoirs, qui serait trop coûteuse pour la plupart des exploitants, serait à réaliser avec l'aide de l'État, par des Coopératives de stockage et de vente, à la formation desquelles tout devrait être mis en cuvre.

Le cas de la Brenne est d'ailleurs loin d'être unique en France; il est simplement typique et c'est pourquoi nous l'avons pris pour exemple.

Dans tout ce qui précède, nous avons considéré que la Carpe devait etre livrée vivante au consommateur; c'est que seule la Carpe vivante peut donner une garantie de fraîcheur. On n'attirera pas la clientèle avec de la Carpe morte refroidie avec de la glace.

Si la Carpe devait étre mise à l'étal, comme la marée, sur un lit de glace, son prix devrait en être sensiblement abaissé.

Quant à la Carpe des gourmets, elle ne saurait être que livrée vivante aux amateurs.

Les diverses mesures que nous préconisons en ce qui concerne l'organisation du marché de la Carpe ne sauraient être mises à exécution que par une action syndicale et coopérative. Déjà la création de viviers d'entrepôts nécessite le groupement des exploitants en syndicats et la constitution de coopératives de vente.

L'aire de ces syndicats (ou des sections syndicales) ne devrait pas etre trop étendue; elle devrait être telle que chaque propriétaire ou exploitant puisse facilement livrer ses poissons à l'entrepôt, en principe, central. 
Ces syndicats ne devraient d'ailleurs pas se borner à constituer des coopératives de stockage et de vente. Ils devraient également avoir dans leurs attributions l'achat en commun des engrais et du matériel (faucardeuses, etc.), ainsi que le contrôle de la qualité des produits, etc.

En tout état de cause, il faut tenir compte de ce que le temps de l'individualisme est passé. Seule compte désormais l'action collective, méthodiquement ordonnée et par conséquent, aux carpiculteurs d'entreprendre eux-mêmes cette action.

Mais, si les carpiculteurs français ont réalisé, à n'en pas douter, des progrès techniques considérables, par contre, ils ont quelque peu négligé le côté commercial de leurs exploitations. Or, c'est du défaut d'organisation de la vente dont ils auront à pâtir un jour, s'ils n'y prennent garde. Il faut d'ailleurs bien considérer que lorsque les circonstances se seront modifiées, lorsqu'en particulier la production se sera accrue grâce à l'utilisation plus totale de races sélectionnées, à l'intensification des faucardages, à l'emploi judicieux et généralisé des engrais, à la mise en valeur d'étangs abandonnés, etc., la carpiculture souffrira sans aucun doute d'une crise sérieuse, si elle ne veille pas dès maintenant à l'avenir, et notamment à l'organisation rationnelle du marché.

Quoiqu'il en soit, nous pensons que par les dispositions analysées dans la présente note, serait établi, du producteur au consommateur, un courant portant uniquement sur de la Carpe de qualité. Ceci ne manquerait pas de provoquer l'augmentation de la consommation et, le cas échéant, la résorption des excédents (ceci dit pour l'avenir), et même l'amélioration des rendements à l'hectare.

Nous pensons donc que c'est dans ce sens qu'il y a lieu de s'orienter.

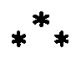

ll y a cependant, pour la Carpe, d'autres possibilités que celles analysées ci-avant.

La Société S. A. F. A. (Société anonyme de Fumaisons alimentaires) a réalisé à Saint-Viatre, dans le Loir-et-Cher, au cours des campages $1942-1943$ et $1943-1944$, le fumage d'un peu plus de 10.000 kilos de Carpes sélectionnées à chaque campagne.

Les produits ont immédiatement bénéficié de la faveur du public. En effet, la chair, pendant la préparation prend une agréable coloration rosée. En outre, le goût caractéristique de fumaison est très apprécié par une nombreuse clientèle.

Ajoutons que la Carpe fumée présente un certain nombre d'autres avantages extrêmement appréciables : grâce à sa durée de conservation, durée qui varie suivant le degré auquel on a poussé la dessication, mais qui peut être de 15 jours par exemple, la Carpe peut être transportée au loin et vendue dans les villages les plus reculés, son transport n'exige 
d'ailleurs que des emballages rustiques et sans glace. Alors que pour expédier 24 kilos de Carpes vivantes par le chemin de fer, il faut 16 kilos de caisses, pour expédier la même quantité de Carpes fumées, il en faut seulement $1 \mathrm{~kg} 200$.

Enfin, bien des ménagères qui répugnent à acheter des Carpes vivantes, pour ne pas avoir à les tuer, font volontiers l'emplette de Carpes fumées. A un autre titre, les Carpes fumées leur donnent satisfaction, car elles peuvent être consommées froides telles quelles ou simplement réchauffées, sans avoir à être vidées.

Toutefois, le prix de revient du fumage de la Carpe est actuellement trop élevé pour être rentable, d'autant plus qu'il faut 2 kilos de Carpes vivantes pour donner 1 kilo de Carpe fumée, la perte en poids au cours de la préparation étant de l'ordre de $49 \%$, provenant pour $25 \%$ de l'enlèvement du tube digestif et des organes annexes, le surplus étant dû à l'évaporation de l'eau des tissus.

Dans les expériences de Saint-Viatre, la Carpe était fumée par le procédé habituel, au bois et à la sciure de bois. Mais, on a pu obtenir ailleurs, ce fumage, par immersion des poissons dans des bains de fuméol, produit extrait des pyroligneux au cours de la carbonisation des bois en vase clos.

Il serait du plus haut intérêt de reprendre ces expériences, en vue de rechercher un abaissement du prix de revient du fumage, et ce d'autant plus que l'emploi de ce procédé ne présenterait pas seulement d'intérêt pour le territoire français, mais aussi pour l'exportation.

En conclusion, pour augmenter, en France du moins, la consommation de la Carpe, il conviendrait d'envisager :

- La création de centres de stockage dans les grands centres de consommation;

- La mise à la disposition des détaillants, de bassins-viviers;

- La production généralisée de Carpes sélectionnées et garanties ;

- L'organisation de viviers de dégorgement, là où les étangs sont répartis entre de nombreux petits propriétaires;

- Enfin, par ailleurs, la reprise des expériences tentées en 1942, 1943 et 1944, en vue d'aboutir, avec un prix de revient raisonnable, au fumage de la Carpe. 